\title{
Modals in Chinese Senior High School Students' Written English
}

\author{
LONG Shaoyun \\ Foreign Languages College, Jiangxi Normal University, Nanchang, China \\ steven8810469@sina.com
}

\begin{abstract}
This study examines the characteristics of modal verbs used in ST2 from CLEC. 2. Methods: keyness analysis: frequency of modal verbs used in ST2 from CLEC are unusually high in comparison with those in A Level from LOCNESS as a reference corpus, producing keywords. 3. Results: only the modal verb must which are introduced early in textbooks is overused by Chinese senior high students while would, could, should, will, can that are introduced comparatively late are underused, which shows that Chinese senior high students seldom use these modal verbs bearing the euphemistic meaning. And the paper gives some interpretations for such phenomena .

Index Terms - modals, overuse and underuse, Chinese senior high school students
\end{abstract}

\section{Introduction}

Modal verbs (Modals)are a type of auxiliary verb that is used to indicate modality. That is, likelihood, ability, permission and obligation (Palmer, F.R., 2001). Modal auxiliary verbs give more information about the function of the main verb that follows it. Although having a great variety of communicative functions, these can all be related to a scale ranging from possibility ("may") to necessity ("must"). Within this scale there are two functional divisions: deontic modality, which is concerned with possibility and necessity in terms of freedom to act (including ability, permission and duty); epistemic modality, which is related to the theoretical possibility of propositions being true or not true (including likelihood and certainty).

Being an important part of English verb system, modals are one of the main media to express modal meanings. Though simple in form, it's quite complex in the aspects of semantics and pragmatics. Besides, modals are universally reflected by ESL/EFL learners and teachers as one of the grammatical points comparatively hard to grasp.

Modals have been studied by a great many of linguistic professionals from different perspectives.

Halliday (1994: 357-358) systematizes these meanings into 'probability', 'usuality', 'obligation' and 'inclination' and further into degrees: 'high', 'median', and 'low'. Palmer (1987) similarly classifies modal meaning 'epistemic', 'deontic' and 'dynamic'. Quirk et al. (1972) use the terms 'ability', 'permission', 'possibility', 'volition', 'obligation', 'probability' and 'logical necessity' among others.

Nowadays, more and more researchers pay their attention to the contrastive study of English native speakers' corpus and non-native English learners' (Coates, 1983).
At the beginning of twenty-first century, contrastive research about the use of modal verbs based on corpus emerged at home (Liu Hua, 2004; Wang Jinquan, 2006; Ma Gang\& Lv Xiaojuan, 2007). There are some other scholars like Liu Wenyan (2009), Long Shaoyun(2011,2012,2013)who did such researches from different perspectives, but all were just limited to college students.

However, the syntactic and semantic characteristics of modal verbs are not given much attention, neither is that section in Chinese senior high students' English written language, and the size of the selected samples in the former researches on modal verbs and modal meaning is rather small and the precision of the result is comparatively low because they are mostly done without nowadays-advanced computer software tools.

\section{Research design}

\section{A. Purpose}

This paper aimed to explore the use tendency of the modals by Chinese senior school students by examining the use pattern in the written form of Chinese senior school students and English native grade 12- to- grade 13 students. The specific questions are :Compared with essays written by senior high school students in Britain, do Chinese senior high school students overuse or underuse modal verbs in English written form? And what are they?

\section{B. Methods}

\section{1) Corpora}

This paper manages to find out the characteristics of modals used in English writing, with CLEC as target corpus and native corpus LOCNESS as reference corpus.

CLEC (Chinese Learner English Corpus)is one of the first learners' English corpus in China, covering about 1 million words of English compositions written by Chinese learners of English with different levels of language proficiency, with their compositions named by ST2, ST3, ST4,ST5and ST6 respectively. This study only concerns ST2, the free English compositions without restriction of time or resource, whose token is 208088.

LOCNESS (Louvain Corpus of Native English Essays) is a corpus of native English essays with a size of 324,304. The data used here are chosen from British pupils' A level essays extracted from LOCNESS, with a token of 60, 209 .

Almost all the essays adopted here are argumentations or expositions which are the writer's idea, values, arguments and 
so on. The writers are all senior students and most of them almost live in the same time. For the purpose of making comparisons, we use the standardized frequency (occurrences per 1,000,000 words) throughout the whole study.

TABLE I. Basic Information of Two Corpora Adopted

\begin{tabular}{|l|l|l|}
\hline \multicolumn{2}{|l|}{ Corpora } & Tokens(per1,000,000 words) \\
\hline Learner corpus & ST2 & 208088 \\
\hline Native corpus & A level & 65841 \\
\hline
\end{tabular}

\section{2) Instruments}

The frequency of modals are concordanced by Antconc and the results are processed in SPSS to analyze the keyness of the target data. CLAWS is also used here to analyze the syntactic and semantic characteristics of modals.

Antconc Tools is a freeware, multi-platform tool for carrying out corpus linguistics research. Here it is used to calculate the strength of keyness of modal sequences in ST2 and A Level. And by comparing the keyness of the modals in two corpora, this study is to examine whether Chinese senior school students have a tendency to overuse or underuse some of the modals .

The author uses Chi-Square Test in SPSS to examine all frequency differences(naming Keyness)between the NS and the NNS corpora with $95 \%$ as the critical level of confidence ( $\mathrm{P}$ value $<.05)$. Here, Keyness, linking two words or phrases usually assumed to be within a given span of each other, is a term used to describe the quality a word or phrase has of being "key" in its context. The set of keywords found in a given text share keyness, and they are co-key. Keyness can be reached through Chi-square in statistics by SPSS and the Log Likelihood Test (Rayson et al. 2004, Oakes 1998). As is shown by the equation of Chi-square(omitted here ), keyness can be plus or minus, indicating over -and -under-use tendency comparing with the natives. And the larger the keyness, the striking the difference.

\section{Keywords Analysis}

Keywords are those whose frequency are unusually high in comparison with some norms. When keyness is very high, and "P" (the probability of the keyness being accidental) is very low, the word can be fairly safely called a keyword. This can be reached by Antconc,using the keyword button.

\section{Results and discussion}

\section{A. The Statistic Frequency of Modal Verbs}

The total number of tokens in target corpus CLEC ST2 is 208088, while that number in reference corpus Locness A level is 65841 . The total number of overall modal verbs in target corpus CLEC ST2 is 3396, while that number in reference corpus Locness A level is 1588, and the log likelihood test value $=156.74, \mathrm{sig}=0.00000$. It can be seen that Chinese senior high students tend to underuse modal verbs.

This phenomenon of underusing modal verbs may be due to the following reasons. The first is that senior high students in China have not been exposed to English modal verbs for enough time, not been receiving adequate training in this aspect. The second is that in English writing process, they are inclined to describe rather than give comment. The third is that being teenagers, their awareness of responsibility and obligation is comparatively less strong than adults.

The frequency of each of the ten modal verbs (will, can, must, could, would, should, may, shall, need, might) is calculated by using the research tool Antconc. Modal verbs such as may, shall, need, might are not frequently used by both Chinese senior high students and British pupils, thus they are not being researched here in this paper.

TABLE II. The frequency of modal verbs of NNS and NS

\begin{tabular}{|l|l|l|l|l|l|l|}
\hline \multirow{2}{*}{ Key words } & \multicolumn{2}{|c|}{ NNS } & \multicolumn{2}{c|}{ NS } & \multirow{2}{*}{ keyness } & \multirow{2}{*}{ P Value } \\
\cline { 2 - 6 } & Freq & $\%$ F & Freq & $\% 0$ & & \\
\hline Will & 956 & 4.59 & 253 & 3.84 & -8.536 & .000 \\
\hline Can & 774 & 3.72 & 276 & 4.19 & -7.894 & .000 \\
\hline Must & 619 & 2.97 & 62 & 0.94 & 66.139 & .000 \\
\hline Could & 427 & 2.05 & 181 & 2.75 & -10.397 & .000 \\
\hline Would & 362 & 1.74 & 432 & 6.56 & -363.529 & .000 \\
\hline Should & 165 & 0.79 & 202 & 3.07 & -78.227 & .000 \\
\hline
\end{tabular}

\section{B. The Underuse of Modal Verbs}

It's shown clearly in Table II that most modal verbs in the learner corpus are significantly different from those in the native corpus by frequence. We can see that the frequency of will is the highest, can is the second, must the third, could the fourth, would the fifth and should the sixth respectively, among which could, would and should, can and will are underused by Chinese senior high students.

The underuse of could and would may be caused by the reason that they are introduced later in English textbooks and as the past tense form of can and will. But actually, could and would are always used in some complex subjunctive conditional sentences to express more complicated and sophisticated modal meaning. Perkins (1983) states that "secondary modals (past form could, would and might) contain much more modal meanings than the primary ones (present form can, will and may)". Not familiar with these two secondary modals, Chinese senior high students feel safe to use more simple primary ones, avoiding the risk of making grammatical mistakes.

The underuse of should is probably connected to Chinese senior high students' physical and psychological status. Being immature teenagers dependent on their parents, they are more used to taking in rather than contributing. And this phenomenon is perhaps having something to do with the culture differences between China and the Western countries.

In Chinese senior high students' mind, NENG is the synonymous equivalence of can. Thus, they prefer to use the modal verb can when they want to express the sense of ability, not so much in the circumstances of permission and possibility. 
The underuse of can is probably caused by Chinese senior high student' informal way of processing English grammatical knowledge.

According to Kennedy (1998), "an analysis of will in a corpus of spoken language (the London-Lund Corpus) and the LOB (Lancaster-Oslo/Bergen Corpus) reveals that it is more frequently used in speech than in writing". Will in English is used to state propositions about the future in the second and third persons but express the willingness, desire or intention in the first person. Let's look at two examples in ST2 in CLEC.

1) You eat just right and do more exercise, you will be healthy.

2) If you do that again, I will hit you.

The first example indicates futurity through the advice the writer gives. And the second example expresses more intention of the writer than futurity. Besides, the style is quite informal.

The use frequency of will by Chinese senior high students is a little lower than that of native speakers at the same age. It may be because the Chinese translation of will is HUI, which is very common in Chinese people's daily conversation.

\section{The Overuse of Modal Verbs}

It can be seen from the above table that one modals are overused, and that is must.

Must is used to express that something is obligatory ("He must leave"). It can also be used to express a prohibition such as "You must not smoke in here", or a resolution such as "I mustn't make that mistake again", or held strong beliefs such as in "It must be here somewhere". Most of the dictionaries and grammar books translate must as "BIXU", which expresses the strong root meaning. When students apply the connotation and principles of "BIXU" in Chinese to the modal verb must, it would lead to the overuse of must. Moreover, it's likely that the overuse of must is caused by the way Chinese senior high students choose to express their thoughts, being direct and rough. They lack the sense of understatement.

The results demonstrate that Chinese senior high students tend to overuse and underuse some modal verbs. To be specific, modal verb must is significantly overused while would is significantly underused.

And the reasons are analyzed as follows:

1) Learners tend to employ the "safe" application of language items. It's true of their use of modal verbs too. They choose to express their thoughts with familiar words, phrases and sentence patterns. So in English writing, they prefer to pick those simplest and easiest ones just to avoid making any grammatical mistakes. And so, it becomes more likely and secure for them to get less minus marks in the composition scoring.

2) The teaching method of modals leads to the overuse and underuse of some modal verbs. Teachers mainly just give the students Chinese translation of modal verbs. However actually, most modal verbs are not equivalent to their corresponding words in Chinese. And teachers have the tendency to instruct learners with the universal use of modals.
Consequently, learners frequently practicing these expressions cause the monotony in their English output .

3) The culture difference between China and Englishspeaking countries also contribute to the significantly divergent use of modal verbs. Native speakers put more emphasis on the objective description of events while Chinese learners are more inclined to posture as the master with strong awareness of responsibility.

\section{Major Findings}

The author attains much useful and reliable information concerning the use of modal verbs in Chinese senior high students' written English. And the characteristics are depicted as follows:

There exists an extremely uneven use frequency of modal verbs in Chinese senior high students' written English. In contrast to essays written by the same level native speakers, Chinese senior high students have been observed overusing modal verbs can, will, and must which are introduced early in textbooks. Meanwhile, they have been observed underusing could, would and should that are introduced comparatively late. This shows that Chinese senior high students rarely use these modal verbs bearing the euphemistic meaning.

\section{Conclusion}

This research is about the use of modal verbs among Chinese senior high students by way of corpus analysis. The sub-corpus ST2 of Chinese Learner English Corpus (CLEC) and the sub-corpus A level of Louvain Corpus of Native English Essays (LOCNEE) are chosen as learner corpus and reference corpus. With the assistance of various software tools, the overused and underused modal verbs have been found out. The possible reasons are illustrated and feasible applications to English language teaching and learning in Chinese senior high school are raised. Still, this study has some limitations that need to be further looked into in future research of this field.

\section{References}

[1] Halliday, M. A. K. An Introduction to Functional Grammar, $2^{\text {nd }}$ ed., London: Arnold /Beijing: Foreign Language Teaching and Research Press, 1994 /2000.

[2] LiuHua, "Modal verbs usage patterns in Chinese English majors," Journal of NingBo University (educational science), vol.5, pp121125.48, May2004.

[3] Kennedy, G. An Introduction to Corpus Linguistics, $1^{\text {st }}$ ed., London: Longman, 1998.

[4] LONG Shaoyun, "A study of modal sequences in English major's argumentations", ChinaEnglisheducation, [EB-OL] http://www.sinoss.net/qikan/2012/0207/12790.html., vol 2, pp.1-13, Dec 2011.

[5] LONG Shaoyun, “The relationship between English major's tendency to use modal sequence and their study time at school",Foreign language education, vol 4, pp.1-12, Dec.2012.

[6] LONG Shaoyun, "A study of modal sequences' grade features in English major's argumentive writing " in Wen. eds. Foreign Language Education in China vol 6. Beijing: Foreign Language Teaching and Research Press, pp. 3-14.Mar.2013.

[7] LIU Wenyan, "A Corpus-Based Study on the Written Form of Chinese English Majors' Use of Modal Sequences”, Changsha, HuNan 
University. 2009.

[8] MA Gang, LVXiao-juan, “ The Analysis on Modal Verbs based on Chinese Learner English Corpus ST6”, CAFLE, vol 6, pp.17-21,Jun. 2007.

[9] Michael, P.Oakes, Statistics for Corpus Linguistics., ${ }^{\text {st }}$ ed., Edinburgh: Edinburgh University Press, 1998.

[10] Palmer, F.R. Mood and Modality.., $1^{s t}$ ed., Cambridge: Cambridge University Press, 2001.
[11] Perkins, Michael R.Modal Expressions in English, $I^{s t}$ ed., Pinter, London, 1983.

[12] Quirk, R., S. Greenbaum, G. Leech \& J. Svartvik. A Comprehensive Grammar of the English Language ., $1^{\text {st }}$ ed., London: Longman, 1985.

[13] Wang Jinquan, "Modal verbs usage patterns in Chinese English learners based on SWECCL". Crazy English Teachers. vol1, pp.44-47, Jan, 2007. 